\title{
Unresolved Problems in the Study of Near-Death Experiences: Some Suggestions for Research and Theory
}

\author{
Kevin J. Drab
}

\section{INTRODUCTION}

The occurrence of unusual experiences in association with neardeath conditions or brief periods of clinical death has attracted widespread attention as a result of the work of researchers such as Moody (1975), Osis and Haraldsson (1977) and Ring (1980). Commonly termed near-death experiences (NDEs) ${ }^{1}$ these events are characterized by their realism and by a common set of features, including out-ofbody experiences, the perception of tunnels, bright lights, landscapes, and deceased friends and relatives, as well as strong positive affect.

Public interest in NDEs has focused on the implication that such cases are evidence for post-mortem survival. However, the majority of scientists studying NDEs and similar phenomena have tended to be more conservative in their outlook, preferring to treat the subject as a psychological issue until further research can "replace present conjectures, if not with certainties, at least with a better judgement than we can now make concerning what is probable" (Stevenson, 1980).

The focus of NDE research is currently on the collection and analysis of anecdotal descriptions. Whether this field of study advances beyond the present level to produce "understanding, prediction and control above the levels achieved by unaided commonsense" (Allport, 1947) may well depend more on the questions asked by researchers than on the nature of the phenomenon itself. A promising beginning has already been made in quantitative and conceptual studies of NDEs (Gabbard, Twemlow \& Jones, in press; Osis \& Haraldsson, 1977; Noyes, 1977; Ring, 1980) which supports the idea that these phenomena will prove amenable to scientific investigation. My hope is that the thoughts and suggestions presented in this paper will contribute to further advance research and theory into NDE phenomena and related subjects.

Although the consensus of most workers in the field is that no satisfactory explanation has yet been found for the NDE, this is not an admission of the failure of scientific method, nor does it imply that a naturalistic explanation will not be found for the phenome- 
non. The investigation of NDEs is still primitive and has yet to develop its own methodology. As a result, most of the limitations and difficulties encountered by NDE researchers are not inherent in the phenomenon itself, but are expressions of problems brought in from other fields. The chief offender here is the field of psychology which, because of more than fifty years of behavioristic orientation, has failed to advance in its study of complex internal events. For example, mental phenomena such as imagery, dreams and thought are as little understood as NDEs. It will take some time before our conceptual tools reach a level of sophistication comparable to our technologies.

In my judgement, the immediate impetus to NDE research will nevertheless come from the application of ideas developed in such disciplines as psychiatry, cognitive psychology, and neurophysiology. As advances are made in these fields, NDE research will benefit and perhaps one day even reciprocate with its own contributions (perhaps as part of a new subspecialty, e.g., psychothanatology).

\section{Guiding Assumptions of NDE Orientations}

A frequent failing in scientific inquiry, and one which could prove particularly harmful to NDE research, is a form of myopia in which an investigator seeking to test a favored hypothesis may be blinded to the occurrence of potentially valuable events not related to that hypothesis (Bachrach, 1965). Progress is far more likely to come from researchers who are receptive to new ideas even when they do not agree with their preconceptions about NDEs.

Although the field of NDE research is still confined to the study of anecdotal reports, no one undertakes an investigation without some assumptions as to "what will happen." Even when these assumptions are implicit or vague, they exert a guiding influence on what one chooses to examine and how that examination is to be conducted.

The assumptions guiding contemporary interest in NDEs can be divided in to the following very general groups:

1. The Parapsychological assumption states that the mind can become independent of the body, and separate from the physical dimension. Since it can function in other realms of existence, it follows that the mind can possibly survive physical death. The similarities between certain eschatological mythologies and NDE descriptions are considered by many as evidence suggestive of an "independent soul."

2. The Psychopsychical assumption has been useful to those para- 
psychologists (Ehrenwald, 1974; Palmer, 1978; Tyrrell, 1953) wishing to explain veridical material gained during out-of-body experiences (OBEs) without subscribing to the idea that the mind can be independent of the body. This approach postulates that the OBE or NDE is a complex tapestry of hallucinatory material and psychicallyderived information. Such an assumption, while leaving the nature of the psychic processes undetermined, accommodates all aspects of the OBE or NDE: the veridical information acquired is attributed to the psi-conducive properties of the condition, and non-veridical contents are simply fantasy-constructs of the unconscious which provide a subjectively compelling "explanation" for the origin of the information.

3. The Archetypal assumption states that a variety of abnormal conditions can activate latent unconscious processes ("matrices") deep in the mind, producing the archetypal elements which constitute NDEs. This view of NDEs has been advocated by such theorists as Jung (1959) and Grof and Halifax (1977) who argue that these neurological substrates of mind are the basis of man's personality and therefore should be investigated to gain a deeper understanding of human nature.

4. The Pathological assumption states that NDEs are the products of a mind disorganized by abnormal psychophysiological conditions. Any regularities in the content of NDEs or suggestions of their transformative effects are explained psychiatrically as ego-defensive maneuvers (e.g., depersonalization, regression, autoscopy, or indiscriminate acceptance of all afferent stimuli) designed to deal with the disrupting condition (Noyes, 1977; Todd \& Dewhurst, 1955).

Because NDEs are such rich experiences and so little understood at present, their features can be "massaged" to prove almost any assumption. The ease with which NDE data can be made to conform to one's guiding assumptions is particularly evident in the proselytizing attempts of some religiously zealous individuals who have published collections of NDE reports claiming to support their particular ideologies (Ford, e.g. 1978; Rawlings, e.g. 1978; Wilkerson, e.g. 1978). An example of the opposite extreme is the argument that the similarities between NDEs and psychopathological states are ipso facto proof that they are identical (Siegel, 1980).

It seems to me that the most sensible position to take on NDEs at this time is that any number of explanations have a fair chance of being correct, and, accordingly, it would be premature to commit oneself to any particular stance beyond its heuristic value for generating hypotheses. There is evidence to suggest that some portions of NDEs may be pathological, while other features may have a 
parapsychological component. It is reasonable to assume that each guiding assumption may have some validity and that the most comprehensive explanation may be that the NDE is an amalgam of pathological, psychological, and parapsychological components which are integrally interlinked in ways that we may never fully understand.

Future research would be far more productive if it is preceded by a consensus on the goals and methodologies of the field, as well as by a concern with clear, operationally oriented definitions. The importance of making premises and assumptions explicit cannot be overemphasized; whenever possible, they should be developed into formal scientific hypotheses. Ring's (1980) effort to develop scales by which to quantify the experiential aspects of this phenomenon is an especially propitious step toward developing reliable and valid measuring instruments in this field. My belief is that the overall aim of NDE research is to gain a publicly useful understanding of the nature of these experiences; therefore, research procedures and theoretical speculations should reflect a concern with elucidating the phenomenon rather than an indiscriminate adoption of concepts and methods from other fields solely for their convenience or prestige value.

It seems to me that a promising direction to follow would be the path of specialization. I believe that considerable value will be found in moving from cumbersome global theories to mini-theories regarding different aspects of the NDE. Later in this paper I will present a number of ideas for such NDE research. Among these suggestions is the possibility that the NDE may actually be a composite of various discrete states of consciousness. If this idea is correct, then each state in the NDE may yield to different forms of investigation, and that in turn should increase the likelihood of interdisciplinary contributions.

For now, however, I wish to begin with some suggestions for improving current approaches to NDE research.

\section{SUGGESTIONS FOR NDE RESEARCH}

\section{Using the Anecdotal Report}

Personal accounts of unusual subjective experiences have never been well received in academic psychology. Should use of the anecdotal report be tolerated in the early stages of inquiry, there is considerable pressure to move as rapidly as possible to testable hypotheses and systematic research. Although the goal of NDE research should be to test such hypotheses in the laboratory, the field of near- 
death studies may have to resign itself to the possibility that anecdotal material will continue to comprise the bulk of its data for some time. Because of the ample supply of NDE reports, however, there should be sufficient data available to develop testable hypotheses.

In view of a continued reliance on anecdotes, researchers should thoroughly examine the issues peculiar to this kind of data collection. Studies of unrestricted descriptions of subjective experiences are generally prone to some of the severest methodological problems existing in the social sciences (Selltiz, Wrightsman \& Cook, 1976).2 Some of these will be considered next.

In view of the strong feelings associated with NDEs, it is understandable that certain individuals may feel compelled to fabricate NDE stories. The media's widespread dissemination of NDE reports provides anyone wishing to describe an NDE or to elaborate upon a meager one all the information necessary to deceive even a seasoned investigator. No satisfactory criteria currently exist by which to evaluate the credibility of an NDE report. The accumulated experience in the field of psychical research regarding psychic fraud and delusion should caution the NDE researcher against accepting an account as true simply because the anecdotist seemed to lack any obvious motive for dishonesty. As West (1948) points out, not only is willful deceit often hard to detect, but sometimes the individual may have no reason other than the pleasure of deception for its own sake, or the desire to foist bogus experiences upon an unappreciative scientific world. Although this problem is somewhat lessened by the agreement among descriptions from individuals ignorant of others' NDEs, there is little question that the validity of any studies carried out on NDEs is a direct function of the honesty of the individuals reporting their experiences.

The unreliability and deceptiveness of memory creates serious problems for many fields concerned with retrospective accounts of experience. The problem of false and distorted memories seems particularly acute when dealing with altered states (Fischer, 1975); once again, however, the agreement among many recalled NDEs makes this difficulty less problematic. The subject of memory difficulties, especially in relation to state-specific knowledge and NDEs, is nevertheless a particularly important one and will be touched on a little later in this paper.

Until the experimental production of NDEs becomes feasible, it is likely that most studies will be based on non-random sampling, i.e., using whatever cases come to one's attention. Since not everyone may wish to communicate his NDE, and the researcher is limited in 
the number of cases he can collect in a geographical area, any sample will necessarily be biased and hence difficult to generalize from.

The possibility of bias of a different sort stems from the researcher's expectations which may prejudice the results of an interview or influence him to reject cases that do not meet his preconception of an NDE. This latter form of selection bias seems to plague all NDE researchers, for one must either draw a line between the vagaries of most hallucinations and those experiences displaying patterns similar to those delineated by Moody (1975), or be overwhelmed by irrelevant material. At this stage of NDE research it is premature to say where to draw the line between acceptable and unacceptable cases for the NDE category. In my own collection, for example, I have accounts of many unusual experiences occurring in connection with perimortem conditions which contain material not relevant to the Moody pattern (e.g., teleological insights, precognitive visions, telepathy, clairvoyance, and so on) but which may nevertheless be important for future study. The Moody pattern offers a general guide, but researchers should avoid inflexibitity here and should remain attentive to miscellaneous elements that may yet open new doors.

One direction of analysis of anecdotal material that might prove especially revealing would be to examine the more qualitative characteristics of the NDE using Marsh's (1977) ideas of focus, structure attributes and flow for describing alternate states of awareness. Another valuable area of investigation would be the use of comparative linguistic analysis to detect differences in the language of NDE reports and other experiences (Landon \& Fischer, 1970).

\section{Classifying the NDE}

Although the term near-death experience is useful when referring to the aggregate of unusual mental events associated with perimortem conditions, it has limited value when discussing the phenomenon in the context of other psychological states. In addition, the lack of an adequate explanation for the NDE makes it difficult to classify within the spectrum of human experience. The emphasis on abnormal physiological conditions often associated with NDEs may further aggravate the problem. Thus one tends to consider the concomitant mental events as pathological in nature. The resultant diversity of terms currently employed to refer to NDEs, e.g., hallucinations, visions, depersonalization, spiritual journeys, and so on, tends to be more evaluative than descriptive and thus may hinder interdisciplinary investigation of the phenomenon.

These difficulties are further compounded by the fact that the 
NDE does not represent a unique set of unusual events; such elements as tunnels, out-of-body experiences, other-worldly beings, light, and panoramic memory, for example, are also found in many other conditions, although recent research suggests that some elements may occur with significantly less frequency in non-perimortem conditions (Drab, in preparation; Gabbard, Twemlow \& Jones, in press). In order to focus on the commonality of these elements across a range of disparate conditions, I have suggested that the term paraversal be used when referring to experiences involving these elements (Drab, 1980). Although this paper is concerned with the NDE, it should be understood that many of the ideas expressed here are equally applicable to the majority of unusual experiences containing paraversal elements. One should, then, view the NDE not as an isolated and special phenomenon in itself but rather as one in a family of related experiences containing paraversal elements. If these experiences share a common mechanism, such an approach will encourage the development of interlocking experiments that will reveal more about the mechanism and how it operates in each situation. A promising direction for future research would be the systematic analysis of similarities and dissimilarities between experiences in perimortem conditions and other conditions associated with unusual states of consciousness brought about by hallucinogenics, temporoparietal tumors, meditation, hypnagogic states, psychomotor epilepsy, $\mathrm{CO}_{2}$ inhalation, lucid dreams, psychotic hallucinations, mystical visions, out-of-body experiences in otherwise normal conditions, and so on.

\section{Undertaking Cross-cultural Studies}

The value of NDEs depends to a great extent on what they may reveal about human nature. Once the reports of near-death experience from other cultures are examined, it will be easier to judge which aspects of the NDE represent more or less universal experiences and which aspects are culturally determined. Cross-cultural studies may also introduce new explanations or insights, especially when a culture has a very different view of NDEs from that which is characteristic of Western Society. Although the establishment of the cross-cultural universality of an NDE element would be a significant advance in understanding, it will not ipso facto prove that the experience is a real event; alternative explanations may be equally supported by such evidence.

The cross-cultural study by Osis and Haraldsson (1977) of hundreds of deathbed experiences of Indians and Americans is so far the 
only investigation of its kind. Although the researchers felt their findings were consistent with those of Moody, regrettably they did not concentrate on most of the more elaborate paraversal elements in their study.

NDE researchers might well take note of a cross-cultural study by Sheils (1978) which demonstrated that about 95 per cent of nearly 70 non-western cultures believed in out-of-body experiences. McIntosh (1980) found results similar to those of Sheils in a field study examining beliefs about out-of-body experiences in three ethnic groups of Papua Guinea. These studies illustrate the type of work NDE researchers could undertake.

Although the attempt to find parallels between NDEs and the eschatological beliefs of other cultures is a valuable avenue for research (Garfield, 1975; Grof \& Grof, 1980; Holck, 1978), it cannot substitute for the collection of first-hand NDE accounts from members of other cultures.

\section{Centralizing Collection of NDE Materials}

It is generally assumed that the more data one collects concerning a particular phenomenon, the fewer problems one has with random sources of error. Therefore, studies based on large samples of NDE reports are as a rule highly preferable to those using few cases. Unfortunately, such studies demand far more time, funds, and facilities than are presently available to most individuals interested in conducting such research. The ideal solution would be to establish a central archives which would serve as a repository for NDE reports. The content of such an archives would be maintained in computer storage, microform, or written material, and could be made available to interested inquirers. The International Association for Near-Death Studies intends to create this type of archives. Toward this end, investigators will be encouraged to submit copies of their own collections for eventual storage. Organizations that frequently receive NDE reports, such as the British and American Societies for Psychical Research and the Psychical Research Foundation, will be asked to send copies of such material to the archives. In order to facilitate retrieval of data, a classification and coding system will need to be developed. Centralization of data collection will also encourage standardization of data gathering methods, through the use of such structured interview schedules as the one developed by Ring (1980). 
Study of Near-Death Experiences

\section{TOWARD A COGNITIVE CONCEPTION OF THE NDE}

\section{Discrete States of Consciousness}

The notion of altered states of consciousness is one of the more attractive conceptions used to classify the NDE. Unfortunately, the term has been used so indiscriminately that it is almost meaningless, having become synonymous with any sort of unusual experience or perception; applying it to the NDE does not clarify the matter. The faddish over use of the altered state idea has not only made it a weak conceptual tool, but has also obscured the important controversy over whether the concept of alterations in consciousness is even valid (Weitzenhoffer, 1978).

Tart $(1975,1978)$ has improved the situation somewhat by proposing the term discrete altered state of consciousness. His use of "discrete state" is intended to single out radical changes in awareness that represent unique, dynamic patterns of structures and subsystems comprising consciousness. Examples of such changes include non-dreaming sleep, dreaming sleep, lucid dreams, marijuana or alcohol intoxication, and meditation. Although there can be considerable variations in the particulars within a discrete state, the overall organization of the state is stabilized by a number of processes causing it to retain a discrete configuration which distinguishes it qualitatively from other states. Regardless of whether altered states are as distinct from each other as Tart suggests, or are merely differences of degree on a continuum of awareness (Horowitz, 1975; Singer, 1977), the general idea helps clarify a muddled issue. As Tart (1977) points out, the common-sense form of disctete states is similar to the gestalt concept of pattern recognition, the feeling that "this condition of my mind feels radically different from some other condition rather than just an extension of it."

The question here, therefore, is whether the NDE can be considered a discrete altered state of consciousness. My initial impression is that the NDE containing elaborate elements such as tunnels, mystical unity, landscapes and panoramic memories exhibits a radical departure from the configuration of ordinary waking consciousness. Closer examination, however, reveals that the NDE does not represent a single unique pattern of consciousness, but rather a variety of discrete configurations. For example, the experience of leaving one's body and floating around the room is clearly a different organization of awareness from the state of ordinary consciousness, but it is also very dissimilar from a state in which one is in a beautiful, brightly illuminated pastoral scene, joyfully interacting with deceased rela- 
tives, or from an overwhelming experience of mystical unity with a brilliant light.

This qualitative difference between contiguous elements may well imply the lack of a direct causal link between them. Therefore, the possibility exists that rather than constituting an unfolding unitary process as Ring (1980) has proposed, the content of NDEs should be regarded as a collection of independent, discontinuous psychological events. At least some of the elements (e.g., out-of-body experiences, mystical unity with light, and being in other-worldly environs) seem to represent discrete states, while the remaining elements (e.g., elevated mood, noises, colors, and simple forms) seem to represent unusual variations in normal cognitive and perceptual processes. Thus, I submit that the NDE is not a specific state of consciousness containing a causally linked sequence of elements, but is instead a condition in which one can expect to find the occurrence of successive, yet independent, discrete altered states of consciousness intermixed with miscellaneous variations in perception, emotion and thought.

This conception places emphasis on the discrete states which are known to occur in other conditions and decreases the importance of the role of the eliciting pathological condition by viewing it as one of a number of situations conducive to the occurrence of such mental events.

\section{Transitional Features of NDEs}

A common feature of NDE reports is the absence of logical transitions between elements. An experient may one moment find himself floating above his body and in the next moment moving through a tunnel or walking in a peaceful meadow, with no sense of how he "went" from one setting to another. Expressions such as "suddenly" and "the next thing I knew" abound in reports used to describe the abrupt changes in elements (Drab, 1976). When interviewing neardeath experients, I have often observed that their descriptions tend to concentrate on continuous aspects such as positive emotions and pay little attention to the lack of continuity between elements-even though such discontinuity in normal waking experience would be rather disturbing to most people.

In a recent study of mine (in preparation) of 71 reports of moving through a tunnel-like space, 79 per cent of the cases experienced no prior elements or sense of transition to "their" tunnels, i.e., they just suddenly were "there". This is particularly surprising in view of the transitional meaning generally given to tunnels and passageways. Of 
the cases studied, only half reported any elements subsequent to their tunnel experiences; and of those reporting further elements, half reported no transition to the new element-only an abrupt change in awareness. Although systematic studies have not yet been performed on other specific features of NDE contents, perusal of the reports in my collection clearly shows the same kind of discontinuity between them. Instances where a logical transition does occur between elements may represent important exceptions to the rule or merely the retrospective interpretations of the experient trying to present his NDE as a cohesive, meaningful event.

These sudden transitions between elements in NDEs may represent shifts from one discrete state to another. Tart (1978) discusses the transitional periods between discrete states and notes that they seem to be fragmentary, frequently being described as a blank space. He postulates that these transitional periods are inaccessible to recall because they represent intervals when consciousness is in a disorganized state; it is at these times that psychological and/or physiological patterning forces are constructing a new discrete state. If this idea is correct, then it may be possible during these transitional periods to detect the formative processes resulting in a new state. Tart suggests that it may be plausible to make thorough observations about these periods by 'training to establish an 'observing self' that phenomenologically is partially or wholly detached from the ongoing operations of consciousness"' (1978).

\section{Language and Memory}

Many NDErs find it difficult to communicate their experiences to others, e.g., Ring (1980) found approximately 60 per cent of his cases claimed that their perceptions and emotional experiences during the near-death episode defied verbal expression. Amother common problem is the NDEr's inability to recall portions of his experience; some experients remember very little, yet have a strong sense that they were aware of far more. Most individuals who undergo perimortem conditions recall no unusual experiences. This finding, however, may be a result of a disruption in memory retrieval rather than a lack of experience per se.

Difficulties in communication and memory are in fact common to many altered states, and create methodological problems for researchers interested in such states. Problems with expression and memory, which may be interdependent, would suggest that the experience is "incommunicable" to the individual's own cognitive structure. This gives it a sense of "specialness" in the individual's mind which may be unwarranted. 
A positive step toward understanding the nature of NDEs is to help experiencers recall and communicate their experiences. The subject of language and memory anomalies is a complex area which has generated some fascinating theoretical models that may apply to NDE research. A brief discussion of these theories may help to illuminate certain aspects of the NDE.

The information-processing approach to hallucinations advocated by Horowitz $(1970,1975)$ assumes that there are various systems used by the mind for the representation of thought: enactive, image and lexical. During many unusual conditions, the mind makes little use of the lexical (language) mode because the image representational system predominates. Thus, in normal consciousness it would be very difficult to translate experience like NDEs into the lexical system. Therefore, it is the over-reliance of the mind on the image mode during altered states, rather than the inadequacy of language itself, which is at fault. Because the typical NDE seems to be composed predominantly of imagery and emotion, this explanation may generate research on how information processing theory can assist us in coping with recall and communication problems. We may be able to obtain this information by encouraging NDErs to communicate through drawings, poetry, and other imagery-oriented media.

Another approach to language and memory difficulties entails the concept of state-boundness which assumes that experiences occurring in a particular state of awareness can never be fully retrieved or communicated accurately in another state. Hence the experience is "bound" or "specific" to that state (Bustamante et al., 1970; Goodwin et al., 1969). In order to regain knowledge acquired during an NDE, the experiencer's original state must be either re-induced or approximated in some symbolic manner. The concept of state-bound experience seems to possess valuable applications for NDE research. For example, the original state may be approximated by hypnotically regressing the individual to the time of the experience or by producing a comparable physiological condition through drugs or external stress.

The attractiveness of the state-bound concept should not, however, lead to a neglect of more mundane explanations for memory and communication difficulties, e.g., repression, inexperience with altered states, poor education or the desire to make the experience seem more profound than it actually was. A state-bound approach also tends to encourage a feeling that many aspects of the NDE are impossible to convey or study in normal consciousness. Tart (1972) has suggested training what he calls state-specific scientists capable of 
conducting research into the states they are experiencing. Although this is an exciting idea, it is questionable whether such investigators will be an actuality soon.

As a variant on the state-bound concept, I propose that we consider the Guthrian idea that information is contiguously paired with whatever pattern of stimuli was present during its acquisition. Retrieval and communication processes are appreciably affected if the stimulating conditions existing at the time of acquisition were altogether different from those existing at the time of attempted retrieval and utilization (Weitzenhoffer, 1954). Conversely, similarity between the original conditions and those present during recall should facilitate retrieval of the original information.

Difficulty in information transfer between states may be due to insufficient similarity between states or to cultural constraints which specify that these states should be experientially separated. Although some altered states are easily repeatable (e.g., through drugs or spiritual disciplines), permitting generalization, the NDE tends to be a single event, and the individual is not always able to assimilate the information adequately. This obstacle may be overcome by exposing individuals who may undergo perimortem conditions in the near future (e.g., chronically ill patients, persons with a high risk of heart attacks, mountain climbers, etc.) to various altered states and by teaching them appropriate language for describing these states (Marsh, 1977; Siegel \& Jarvik, 1975). This "cross-training" approach may give the individuals a wider associative range of patterns that will assist recall and communication of NDEs if they do occur.

Psychophysiological Correlates of NDEs

Assuming a direct relationship exists between psychological and physiological events, it would seem reasonable to look for specific psychophysiological indices of the NDE as a distinct state or composite of states. Palmer and Vassar (1974), Sarbin and Slagle (1972), and Kelly (1977) have argued that the weaknesses of verbal reports can be alleviated to some degree by combining them with psychophysiological measures as convergent indices of a particular experience. Although there are considerable difficulties in assessing the relationship between subjective states and physiological processes (Kennedy, 1976), any progress in this area could mark a breakthrough in NDE research. If a certain psychophysiological pattern were found to accompany the NDE, this knowledge could rapidly lead to fundamental insights into the structure and operation of the mechanisms producing the experience. For those who may feel 
resistant to reducing the NDE to a physiological explanation, it is well to remember that we are only discussing the conditions that are conducive to a variety of experiences containing paraversal elements. Controlled study of NDEs may be accomplished if individuals can be trained, possibly through biofeedback, voluntarily to produce relevant physiological states in themselves.

\section{CONCLUSIONS}

There is a tendency to forget that however unusual an experience may be, it still involves a functioning mind that continues to receive, interpret, store and retrieve information often in the same ways used in its normal state. The NDE is, therefore, a cognitive phenomenon regardless of its nature; the NDEr retains a human mind attempting to make sense of the information presented. Even if the parapsychological explanation proves correct, there is no reason to assume that a disembodied mind ceases to organize and use information in ways consistent with everyday cognitive processing.

This assumption has influenced most of what I have discussed in this paper and suggests that some of the more experimentally productive approaches to the NDE will be based on what is already known about normal cognitive functions.

In any event, the subject of NDEs is an exciting and challenging area which will benefit from the contributions of both the layman and the scientist. Indeed, at this stage in NDE research, no idea is too outlandish if it stimulates rational inquiry.

In this paper, I have discussed only a few of the new directions that NDE research might take and have outlined a cognitive conception of the NDE which I believe merits further development. I hope that my suggestions will contribute to the growing interest in the interdisciplinary scientific study of the NDE.

\section{NOTES}

1. My own preference would be for the more inclusive phrase perimortem experiences.

2. Since many of these problems are created by individual idiosyncracies the strong interagreement among NDE descriptions tends to improve the situation slightly.

\section{REFERENCES}

Allport, G. W. The use of personal documents in psychological 
science. N.Y.: Social Science Research Council, 1947.

Bachrach, A. J. Psychological research: an introduction. N.Y.: Random House, 1965.

Bustamante, J., Jordan, A., Vila, M., Gonzales, A., \& Insu, A. State dependent learning in humans. Physiology and Behavior, 1970, 5, 793-796.

Drab, K. A preliminary study of some Theta Experience reports. Unpublished research summary, 1976.

Drab, K. Researcher suggests broader classification for near-death phenomena. Anabiosis, 1980, 2, 6.

Drab, K. Tunnel experiences-a study of their occurrence in various altered states of awareness. In preparation.

Ehrenwald, J. Out-of-the-body experiences and the denial of death. Journ. Nervous and Mental Disease, 1974, 159, 227-233.

Fischer, R. On the remembrance of things present. Journ. of Altered States of Consciousness, 1975-6, 2, 361-369.

Ford, M. On the other side. Plainfield, N.J.: Logos International, 1978.

Gabbard, G., Twemlow, S., \& Jones, F. Do near-death experiences occur only near death? Journ. Nervous and Mental Disease. (In press.)

Garfield, C. A. Consciousness alteration and fear of death. Journ. of Transpersonal Psychol, 1975, 7, 147-175.

Goodwin, D., Powell, B., Bremer, D., Hoine, H., \& Stern, J. Alcohol and recall: state dependent effects in man. Science, 1969, 163, 1358-1360.

Grof, S., \& Halifax, J. The human encounter with death. N.Y.: E. P. Dutton, 1977.

Grof, S., \& Grof, C. Beyond death: the gates of consciousness. N.Y.: Thames and Hudson, 1980.

Holck, F. H. Life revisited (parallels in death experiences). Omega, 1978, 9, 1-11.

Horowitz, M. J. Image formation and cognition. N.Y.: AppletonCentury-Crofts, 1970.

Horowitz, M. J. Hallucinations: an information-processing approach. In R. K. Siegel \& L. J. West (Eds.) Hallucinations: behavior, experience and theory. N.Y.: Wiley \& Sons, 1975.

Jung, C. G. Synchronicity: an acausal connecting principle. The structure and dynamics of the psyche. Princeton, N.J.: Princeton University Press, 1959.

Kelly, E. F. Psychophysiological correlates of PSI processes. Parapsychology Review, 1977, 8, 1-9.

Kennedy, J. E. A methodological note on psychophysiological 
studies in parapsychology. Journ. Parapsychology, 1976, 40, 308-310.

Landon, G. M., \& Fischer, R. On common features of the language of creative performance and hallucinogenic drug-induced creative experience. In W. Keup (Ed.), Origin and mechanisms of hallucinations. N.Y.: Plenum Press, 1970. 413-433.

Marsh, C. A framework for describing subjective states of consciousness. In N. E. Zinberg (Ed.), Alternate states of consciousness. N.Y.: Macmillan, 1977.121-144.

McIntosh, A. E. Beliefs about out-of-the-body experiences among the Elema, Gulf Kamea and Rigo peoples of Papua New Guinea. Journ. Soc. for Psychical Res., 1980, 50, 460-478.

Moody, R. Life after life. Atlanta, Ga.: Mockingbird Books, 1975.

Noyes, R., \& Kletti, R. Depersonalization in response to life-threatening danger. Comprehensive Psychiat., 1977, 18, 375-384.

Osis, K., \& Haraldsson, E. At the hour of death. N.Y.: Avon, 1977.

Palmer, J. The out-of-body experience: a psychological theory. Parapsychology Review, 1978, 9, 19-22.

Palmer, J., \& Vassar, C. ESP and out-of-the-body experiences: an exploratory study. Journ. of the Amer. Soc. for Psychical Res., $1974,68,257-280$.

Rawlings, M. Beyond death's door. N.Y.: Thomas Nelson, Inc., 1978. Ring, K. Life at death. N.Y.: Coward, McCann \& Geoghegan, 1980.

Sarbin, T. R., \& Slagle, R. W. Hypnosis and psychophysiological outcomes. In E. Fromm \& R. E. Shors (Eds.), Hypnosis: research development and perspectives. Chicago: Aldine-Atherton, 1972. 185-214.

Selltiz, C., Wrightsman, L., \& Cook, S. Research methods in social relations. (3rd ed.) N.Y.: Holt, Rinehart and Winston, 1976.

Sheils, D. A cross-cultural study of beliefs in out-of-the-body experiences, waking and sleeping. Journ. Soc. for Psychical Res., 1978, $49,697-741$.

Siegel, R. K., \& Jarvik, M. E. Drug-induced hallucinations in animals and man. In R. K. Siegel \& L. J. West (Eds.), Hallucinations: behavior, experience and theory. N.Y.: Wiley \& Sons, 1975. 81-161.

Siegel, R. K. The psychology of life after death. American Psychologist, 1980, 35, 911-931.

Singer, J. L. Ongoing thought: the normative baseline for alternate states of consciousness. In N. E. Zinberg (Ed.), Alternate states of consciousness, N.Y.: Macmillan, 1977, 89-120.

Stevenson, I. Comments on The reality of death experiences: a personal perspective. Journ. Nervous and Mental Disease, 1980, 168, 271-272. 
Tart, C. T. States of consciousness and state-specific sciences. Science, 1972, 176, 1203-1210.

Tart, C. T. States of consciousness. N.Y.: Dutton, 1975.

Tart, C. T. Putting the pieces together: a conceptual framework for understanding discrete states of consciousness. In N. E. Zinberg (Ed.), Alternate states of consciousness. N.Y.: Macmillan, 1977. 158-219.

Tart, C. T. Altered states of consciousness: putting the pieces together. In A. Sugerman \& R. Tarter (Eds.), Expanding dimensions of consciousness. N.Y.: Springer, 1978. 58-80.

Todd, J., \& Dewhurst, K. The double: its psychopathology and physiology. Joum. Nervous and Mental Disease, 1955, 122, 47-55.

Tyrrell, G. N. M. Apparitions. The seventh Frederic W. H. Myers Memorial lecture. London: Duckworth, 1953.

Weitzenhoffer, A. M. The influence of hypnosis on the learning process: some theoretical considerations. Joum. of Clin. \& Experimental Hypnosis, Part 1, 1954, 2, 191-200; Part 2, 1955, 3, 148-165.

Wietzenhoffer, A. M. Hypnotism and altered states of consciousness. In A. Sugerman \& R. Tarter (Eds.), Expanding dimensions of consciousness. N.Y.: Springer, 1978. 183-225.

West, D. J. The investigation of spontaneous cases. Proc. of the Soc. for Psychical Res., 1948, 8, 264-300.

Wilkerson, R. Beyond and back. N.Y.: Bantam Books, 1978.

Requests for reprints to:

Kevin Drab

24 Ship Street

Hingham, Massachusetts 02043 